\title{
Corpus callosotomy performed with laser interstitial thermal therapy
}

\author{
Jarod L. Roland, MD, ${ }^{1}$ Syed Hassan A. Akbari, MD, ${ }^{2}$ Afshin Salehi, MD, MS, ${ }^{2}$ and \\ Matthew D. Smyth, MD2
}

\begin{abstract}
${ }^{1}$ Department of Neurological Surgery, University of California, San Francisco, California; and ${ }^{2}$ Department of Neurological
\end{abstract} Surgery, Washington University in St. Louis, St. Louis, Missouri

\begin{abstract}
OBJECTIVE Corpus callosotomy is a palliative procedure that is effective at reducing seizure burden in patients with medically refractory epilepsy. The procedure is traditionally performed via open craniotomy with interhemispheric microdissection to divide the corpus callosum. Concerns for morbidity associated with craniotomy can be a deterrent to patients, families, and referring physicians for surgical treatment of epilepsy. Laser interstitial thermal therapy (LITT) is a less invasive procedure that has been widely adopted in neurosurgery for the treatment of tumors. In this study, the authors investigated LITT as a less invasive approach for corpus callosotomy.
\end{abstract}

METHODS The authors retrospectively reviewed all patients treated for medically refractory epilepsy by corpus callosotomy, either partial or completion, with LITT. Chart records were analyzed to summarize procedural metrics, length of stay, adverse events, seizure outcomes, and time to follow-up. In select cases, resting-state functional MRI was performed to qualitatively support effective functional disconnection of the cerebral hemispheres.

RESULTS Ten patients underwent 11 LITT procedures. Five patients received an anterior two-thirds LITT callosotomy as their first procedure. One patient returned after LITT partial callosotomy for completion of callosotomy by LITT. The median hospital stay was 2 days (IQR 1.5-3 days), and the mean follow-up time was 1.0 year (range 1 month to 2.86 years). Functional outcomes are similar to those of open callosotomy, with the greatest effect in patients with a significant component of drop attacks in their seizure semiology. One patient achieved an Engel class II outcome after anterior two-thirds callosotomy resulting in only rare seizures at the 18-month follow-up. Four others were in Engel class III and 5 were Engel class IV. Hemorrhage occurred in 1 patient at the time of removal of the laser fiber, which was placed through the bone flap of a prior open partial callosotomy.

CONCLUSIONS LITT appears to be a safe and effective means for performing corpus callosotomy. Additional data are needed to confirm equipoise between open craniotomy and LITT for corpus callosotomy.

https://thejns.org/doi/abs/10.3171/2019.9.JNS191769

KEYWORDS corpus callosotomy; laser interstitial thermal therapy; MR-guided LITT; epilepsy

$\mathrm{N}$ EUROSURGICAL treatment of medically refractory epilepsy (MRE) is an effective option for carefully selected patients. Numerous studies, including several randomized controlled trials, have described the benefit of surgical intervention over continued medical management. . $7,35^{7}$ Despite such encouraging data, the majority of eligible patients never receive surgical evaluation. ${ }^{8}$ One potential factor contributing to this discrepancy is the perception of morbidity associated with open cranial surgery by patients, families, and their referring physicians. Therefore, developing procedures that reduce morbidity while attaining similar efficacy may increase our ability to impact the treatment of MRE.
Corpus callosotomy is a palliative procedure that is effective at reducing seizure burden in patients with multifocal and generalized MRE, with the greatest specific benefit achieved in reducing atonic seizures (drop attacks). Because drop attacks occur suddenly, and typically without warning, they are often associated with significant injuries. Disconnecting the corpus callosum (CC) limits the rapid spread of a generalized seizure onset and thereby reduces the rate of drop attacks and their associated injuries. In this manner, corpus callosotomy can provide a meaningful reduction in the severity and adverse effects of seizures even without necessarily reducing seizure frequency.

ABBREVIATIONS CC = corpus callosum; DWI = diffusion-weighted imaging; FC = functional connectivity; iMRI = intraoperative MRI; LITT = laser interstitial thermal therapy; MRE = medically refractory epilepsy; rs-fMRI = resting-state functional MRI; TDE = thermal damage estimate.

SUBMITTED June 25, 2019. ACCEPTED September 30, 2019.

INCLUDE WHEN CITING Published online December 13, 2019; DOI: 10.3171/2019.9.JNS191769. 
Corpus callosotomy is traditionally performed via open craniotomy with interhemispheric microdissection to divide the CC. The CC can be divided in its entirety from rostrum to splenium in one setting, or only partially divided such as the anterior two-thirds with the splenium spared. There is evidence for better outcomes with complete callosotomy. ${ }^{9}, 13,16$ However, partial callosotomy may carry lower risk of a disconnection syndrome, particularly in patients with high cognitive function, and the option to return for a second-stage completion of the callosotomy remains. ${ }^{2}$

Craniotomy for corpus callosotomy is not without morbidity. Risks are similar to those of most other cranial procedures and include hemorrhage, infection, injury to adjacent cerebral structures, frontal lobe retraction, venous ischemia and infarction, postoperative CSF leakage, development of hydrocephalus, poor wound healing, and need for repeat surgery. Morbidity also includes pain and missed days of productivity. With the goal of reducing these risks and morbidity, many surgeons have begun using less invasive alternatives. Examples include wider adoption of stereo-EEG for intracranial monitoring, laser ablation for localized epilepsy, and endoscopic functional hemispherotomy for hemispheric seizure onset ${ }^{1,10,33}$ (see the recent review by Roland and Smyth ${ }^{27}$ ). A less invasive procedure for generalized epilepsy is similarly desirable.

Laser interstitial thermal therapy (LITT) is an FDAapproved method to necrotize or coagulate soft tissue in the brain and other organs. It is performed with the guidance of an advanced MRI method for real-time thermography. ${ }^{38}$ During LITT treatment, a gradient recalled echo sequence is continuously acquired, from which the proton resonance frequency shift is measured. Deviation from a baseline temperature is calculated by detecting change in the phase angle of proton resonance frequency shift signal from a reference image. ${ }^{24}$ This calculation is performed in real time to measure the thermal heating applied by the surgically implanted laser fiber. By integrating the heat delivered over time, an estimate of tissue destruction is calculated according to the Arrhenius equations for the temperature dependence of chemical reactions..$^{20,38}$ The results of this model are then transformed into a thermal damage estimate (TDE) and presented to the surgeon over anatomical images to guide the extent of ablation. The commonly used term "MRI-guided LITT" refers to the ability to guide the laser ablation by observing the TDE in real time. In this text, we refer to this modality simply as LITT.

LITT has been most widely adopted in neurosurgery for the treatment of tumors. ${ }^{20,32}$ It has also been applied to mesial temporal lobe epilepsy $y^{10,11,34,36}$ and localized focal epilepsy. ${ }^{19,37}$ Few studies have suggested that LITT may be effective in performing corpus callosotomy with acceptable risk and reduced surgical morbidity. Here, we present our efforts in the continued innovation of less invasive neurosurgical treatment with a retrospective case series of corpus callosotomy performed with LITT in 10 patients at our institution.

\section{Methods}

We retrospectively reviewed all patients who under- went LITT for ablation of the CC for treatment of MRE at St. Louis Children's Hospital. Patients were included if the target for ablation was any portion of the $\mathrm{CC}$, including a residual from a prior partial corpus callosotomy or hemispherotomy, partial agenesis of the CC, or a structurally normal CC. The institutional review board at Washington University in St. Louis approved the study for retrospective review of clinical records with a waiver of written consent.

The patients in this series were identified as candidates for corpus callosotomy by a multidisciplinary epilepsy review committee. Candidacy for corpus callosotomy was determined by clinical criteria alone. The senior author (M.D.S.) offered open craniotomy versus LITT for callosotomy if both procedures were deemed acceptable. Factors considered for LITT to be an acceptable option included favorable CC morphology such as a thick and flat body, no prior implant prohibiting fiber placement, and ability to obtain an MRI scan under general anesthesia. For our initial experience, we opted to limit upfront LITT treatments to partial callosotomy that could be completed with 2 fibers. Therefore, we also preferred open craniotomy for cases in which complete callosotomy was desired in a single procedure.

We planned trajectories on preoperative high-resolution MRI with gadolinium contrast and CT with contrast. We also used resting-state functional MRI (rs-fMRI) to localize the cortical sensorimotor network as well as diffusion tensor imaging to identify the corticospinal tract in white matter. This technique was described in our prior work in pediatric patients ${ }^{25}$ as well as across our institution. ${ }^{18} \mathrm{We}$ previously compared resting-state sensorimotor network mapping in pediatric epilepsy patients ${ }^{26}$ as well as adult epilepsy ${ }^{21}$ and brain tumor ${ }^{5}$ patients. For all cases we used the ROSA (Zimmer Biomet) robotic navigation system for planning and placement. We used a 6-mm virtual fiber diameter to ensure that no vessels crossed this safe zone.

After select procedures, we analyzed rs-fMRI data to qualitatively support functional disconnection of the hemispheres. Our group recently reported a detailed study on the role of the CC in interhemispheric functional connectivity (FC). ${ }^{28}$ The analysis here is carried out in the same fashion. The rs-fMRI data were acquired using an echo-planar imaging sequence sensitive to the blood oxygen level-dependent contrast with TR $2070 \mathrm{msec}$, TE 25 msec, flip angle $90^{\circ}$, and voxel size $4.0 \times 4.0 \times 4.0 \mathrm{~mm}$ in 2 runs of 200 frames, totaling approximately 14 minutes. We used a 3T Siemens Trio MRI scanner for all functional imaging. We performed typical preprocessing procedures as previously described. ${ }^{28,29}$ We computed FC between a 6-mm spherical seed region and every other voxel as the Pearson correlation coefficient and then applied the Fisher z-transform.

\section{Surgical Procedure}

We placed the laser fibers in our operating room under sterile conditions. The patient was positioned supine on the operating table with the head in a neutral or slightly turned position. If a posterior fiber trajectory was used, the head was turned lateral to the opposite side. The patient's head was immobilized with either a Mayfield skull clamp 
or Leksell frame (Fig. 1A) and then secured to the ROSA robotic system.

For case 1, laser tracing was used for stereotactic coregistration. In the remaining cases, either bone fiducials or the Leksell frame with a custom reference (Fig. 1B) was used for stereotactic coregistration. When using bone fiducials or the Leksell frame, these were placed immediately after induction of anesthesia, and then a high-resolution CT scan was obtained and aligned to the preoperative MRI scan.

The ROSA robot was then used to mark the entry point for each fiber. A small amount of hair was clipped, and the field was prepped and draped in a sterile fashion. We did not place an incise drape over the skin to avoid it being caught in the drill or bone anchor during fiber placement.

We infiltrated the local anesthetic and made a stab incision at the entry point. We then used the ROSA robotic arm with a 3.4- $\mathrm{mm}$ guide tube to direct a handheld power drill with a 3.2-mm bit. A depth stop was set to the depth measured on the preoperative CT. We then passed a dural dilator down the tract to coagulate and pierce the dura by applying monopolar electrocautery to the proximal end of the dilator. Between each step we irrigated the wound to clear debris.

The ROSA arm was then used to measure a distance from the tip of the bone anchor to the depth of the target (Fig. 1C). The measurements were marked on the cooling catheter with an adhesive strip folded lengthwise. First, the outer cooling catheter with a stiffening stylet was placed down the tract of the anchor to the target depth. Then the stiffening stylet was removed and the laser fiber was passed and secured. If more than 1 fiber was used, this procedure was repeated and each fiber clearly labeled.

We then removed the drapes (Fig. 1D) and transferred the patient to the MRI unit. For case 1, the procedure was performed in an intraoperative MRI (iMRI) suite with the Monteris NeuroBlate system; therefore, no travel was required.

Ablation began at the depth of the fiber trajectory. MRI thermometry was used to follow the TDE. Once the desired area was treated at the initial depth, the fiber was withdrawn by approximately $5-8 \mathrm{~mm}$, and ablation continued in a similar fashion. Because the TDE margins extend radially from the laser source, the treatment zone is generally spherical. Each successive treatment zone was overlapped to avoid intervening valleys of potentially incomplete treatment. In this fashion, we stacked successive treatments to create a cylindrical treatment volume along the length of the $\mathrm{CC}$ (Video 1).

VIDEO 1. Video showing intraoperative thermography and thermal damage estimates while performing an anterior two-thirds corpus callosotomy. The video begins with ablation at the distal body fiber and progresses anteriorly. Then we switch to the second fiber to ablate the rostrum and genu to complete the anterior callosotomy. The left frame shows real-time thermometry and the right frame shows the calculated thermal damage estimates. Copyright

Washington University in St. Louis. Published with permission. Click here to view.

Standard LITT technique was used to ensure safe tissue heating. This included safety checks for temperature thresholds at adjacent structures. We did observe heat

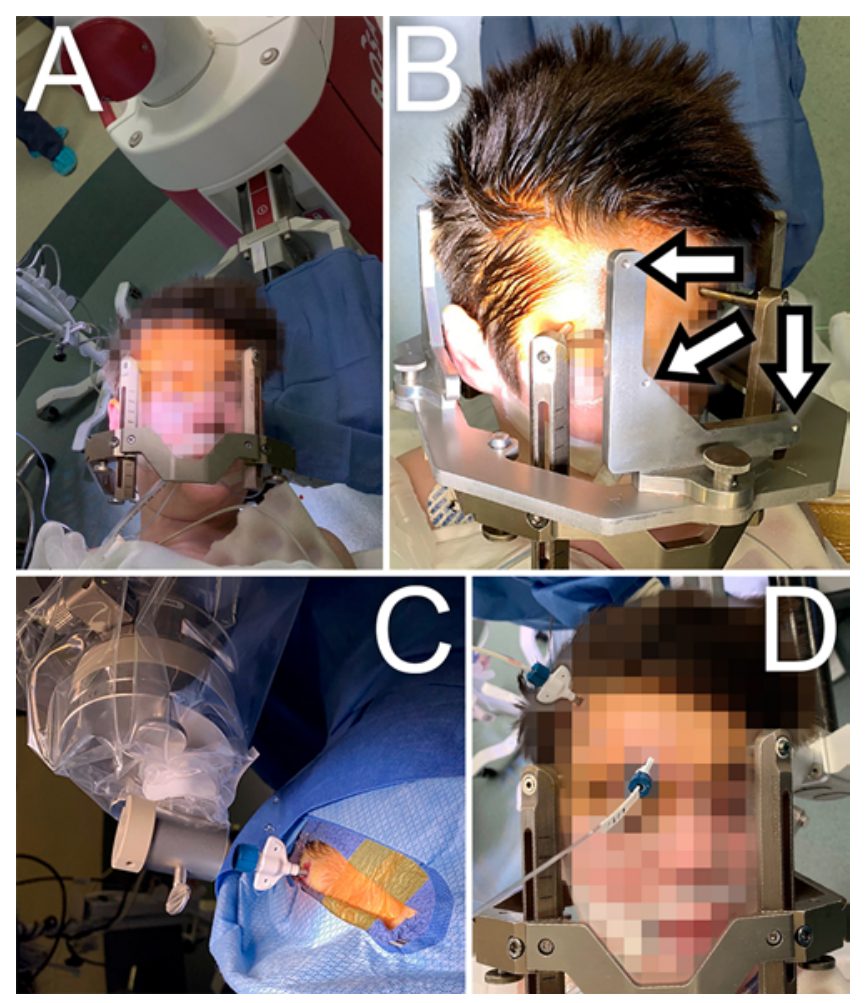

FIG. 1. Surgical setup. A: The patient's head is secured in 4-point fixation with the Leksell frame, which is then fixed to the ROSA robotic system. B: A custom-configured reference template is attached to the Leksell frame for registration with 3 predefined points on each of the right, left, and anterior vertical reference plates (arrows). C: The patient is then draped, and each anchoring bolt and laser fiber is placed. D: At completion, the drapes are removed with care to not displace the laser fibers. Figure is available in color online only.

sinking from CSF spaces, such as the ventricle, as is expected for LITT.

After satisfactory ablation, we performed anatomical imaging to confirm the extent of treatment. ${ }^{14} \mathrm{We}$ first administered gadolinium and then performed diffusionweighted imaging (DWI) followed by T1-weighted MPRAGE. The time required for DWI was sufficient for adequate contrast uptake on the subsequent T1-weighted image. The DWI sequence identified diffusion restriction corresponding to the treatment zone. The contrasted T1weighted image identified enhancement at the margin of the treatment zone. If any area of insufficient ablation was identified, we repositioned the fiber to the desired location and extended treatment.

Once adequate ablation was confirmed, the patient was transported back to the operating room for closure. The anchors and any bone fiducials were removed and closed with a single monofilament suture. We administered weight-based dexamethasone for approximately 48 hours after the procedure and was stopped if the patient recovered well.

\section{Results}

Ten patients underwent 11 LITT procedures for corpus callosotomy. We began our experience with procedures 

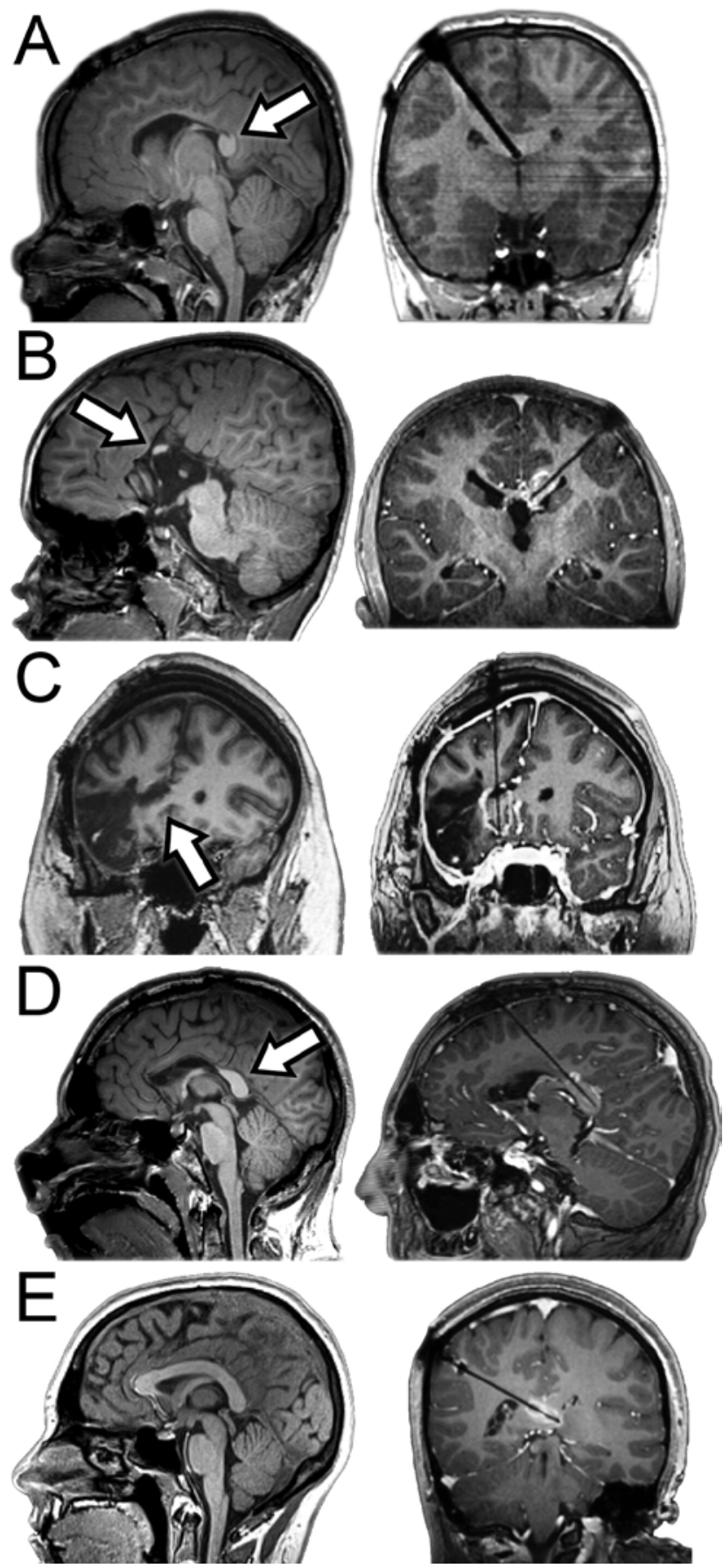

FIG. 2. Single-fiber procedures. Preoperative and intraoperative T1weighted MR images of a corpus callosotomy performed with LITT that required only a single laser fiber. A: Case 1 with a residual splenium (arrow) of the CC (left, sagittal view) and the location of a Monteris laser fiber for ablation (right, coronal view). B: Case 2 with a partial agenesis (arrow) of the CC (left, sagittal view) and a single laser fiber used to ablate this focal corpus callosum (right, coronal view). C: Case 3 demonstrates a remnant of the genu of the corpus callosum (arrow) after functional hemispherotomy (left, coronal view) and a single laser fiber to ablate this residual connection (right, coronal view). D: Case 4 with a residual splenium (arrow) of the CC (left, sagittal view) and location of a single laser fiber for ablation (right, sagittal view). E: Case 11 with a normal CC (left, sagittal view) and a single laser fiber for ablation of the splenium only in a patient with light-induced photic seizures with parietooccipital synchrony on electroencephalogram (right, coronal view). that could be completed with placement of a single fiber (Fig. 2). Collectively, we performed 5 procedures with a single fiber. Two were for completion of a prior open partial corpus callosotomy, 1 for ablation of a residual at the rostrum of the $\mathrm{CC}$ after functional hemispherotomy, 1 for complete ablation of partial agenesis of the $\mathrm{CC}$, and 1 for focal ablation of only the splenium.

We then began performing anterior two-thirds partial corpus callosotomy with a 2-fiber configuration (Fig. 3). The general configuration of the 2-fiber approach is for one fiber along the length of the body of the CC, primarily in the axial plane, and a second fiber through the genu into the rostrum, in an oblique coronal plane. Five patients received anterior two-thirds LITT callosotomy upfront.

For our first anterior two-thirds callosotomy (case 5), we used a posterior approach to the body of the CC. At the beginning of the LITT phase, we discovered that the body fiber was off target, into the lateral ventricle, then back into the anterior body (Fig. 3A). We ablated the extent achievable with this placement and then repositioned the fiber in the operating room. However, even after repositioning and repeating ablation, a segment of the body was left untreated (Fig. 4A). Following unsatisfactory seizure response, this patient returned for a second procedure (case 7) for completion of the callosotomy by placing 1 fiber in the missed segment (Fig. 4B) and 2 additional fibers in the remaining posterior portion of the CC (Fig. 4C and D).

For this patient, we analyzed rs-fMRI data after partial callosotomy in case 5 and after completion in case 7 to qualitatively support effective functional disconnection. We observed a dramatic decrease in interhemispheric FC after the complete callosotomy (Fig. 5). This is contrasted to residual connectivity after the partial callosotomy, which is consistent with our previously published work. ${ }^{28}$

We found the posterior approach to be suboptimal due to its length (10.6 cm from target to the skull outer table) and difficult head position required to avoid the anchor bolt and fiber colliding with the MRI table. For subsequent procedures we used an anterior approach for the body fiber due to its shorter trajectory length (mean $9.3 \mathrm{~cm}$, range $8.7-9.7 \mathrm{~cm}$ ), with the trade-off of a less cosmetic location for the stab incision at the eyebrow (Fig. 1). This anterior approach is unique among configurations in existing literature. For fibers placed in the genu, the entry point was behind the hairline and had a mean length of $6.2 \mathrm{~cm}$ (range $5.0-7.0 \mathrm{~cm}$ ).

Our first procedure (case 1) was performed using the NeuroBlate (Monteris Medical) system integrated in an IMRIS (Minnetonka) iMRI suite (Fig. 2A). The subsequent 10 procedures were performed with the Visualase (Medtronic Inc.) system within a diagnostic MRI suite.

The median hospital stay was 2 days (IQR 1.5-3 days) (Table 1). One outlier was an inpatient for 6 days (case 5) related to ongoing seizures and emesis. The operative time was generally longer than that for a typical open callosotomy procedure. The median operating room time was 6.9 hours (IQR 5.12-7.07 hours). One outlier took 9.5 hours of operating room time due to a technical complication where the saline cooling circuit leaked on the MRI headcoil controller circuit board, rendering the MRI inoper- 

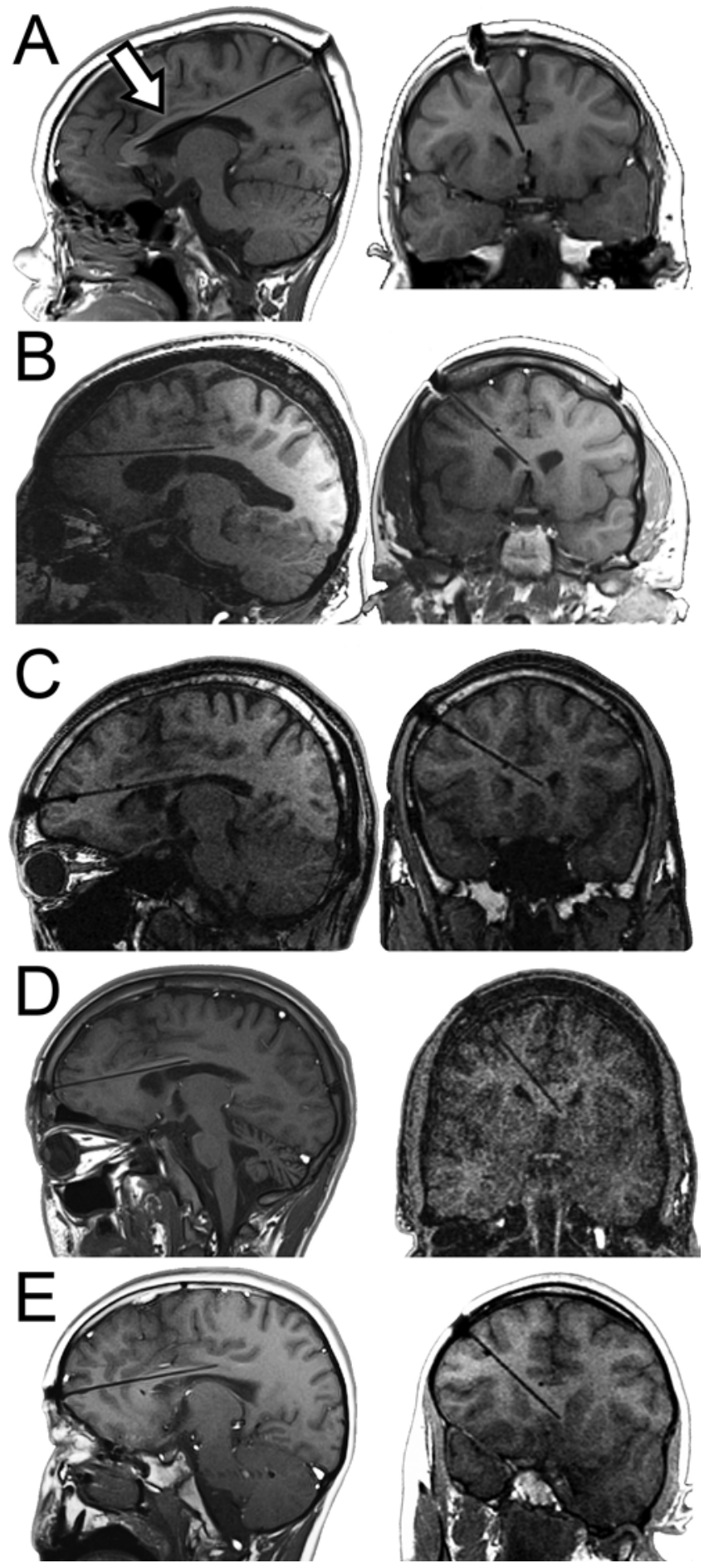

FIG. 3. Two-fiber anterior two-thirds callosotomy. Intraoperative sagittal (left) and coronal (right) T1-weighted MRI scans are shown for anterior two-thirds corpus callosotomy performed with LITT using a 2-fiber configuration. For all cases the body fiber is shown on the left and the genu fiber is shown on the right. A: Case 5 was performed with a posterior approach to the CC body and was complicated by aberrant targeting (arrow) resulting in a portion of the body being missed. For subsequent procedures (cases $6[B], 8[C], 9[D]$, and $10[E]$ ), the body laser fiber was placed from an anterior trajectory.
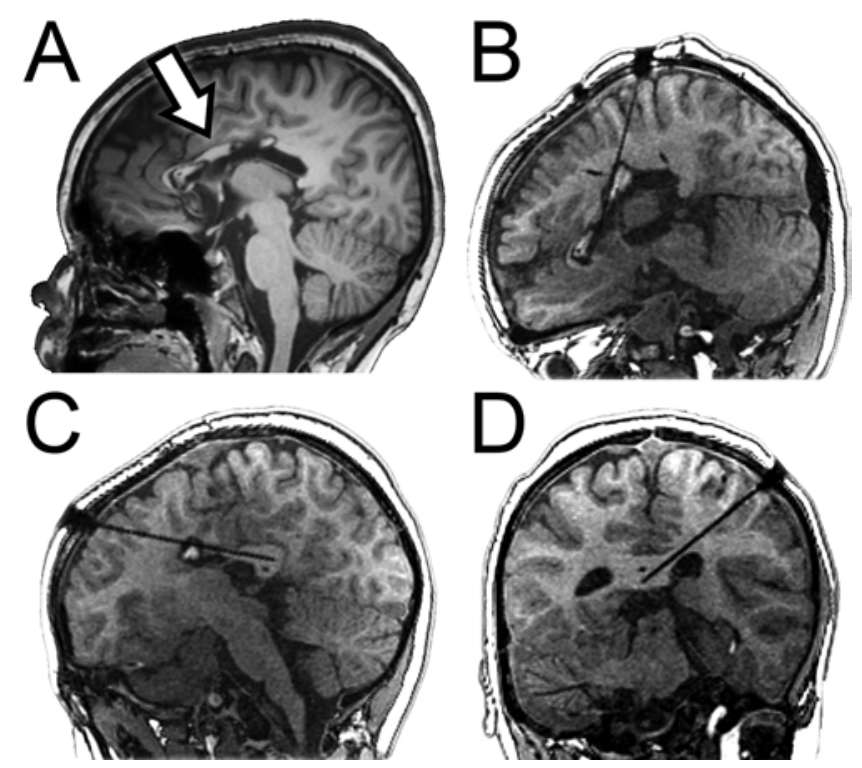

FIG. 4. Fiber configuration for completion of a prior subtotal ablation. The patient initially treated in case 5 (A) had a segment of the body of the CC that was not ablated (arrow) by the first procedure due to aberrant fiber placement. The patient returned in case 7 for completion of the callosotomy with 1 fiber placed to treat the anterior segment (B) and 2 additional fibers for the posterior body (C) and splenium (D).

able. We mobilized the Visualase system to another diagnostic MRI unit and completed the procedure.

Hemorrhage occurred in 1 patient (case 4) along the proximal fiber trajectory that was placed through the bone flap of a prior open partial callosotomy. The supplementary motor area hemorrhage was not present on intraoperative imaging immediately after ablation but was noted on postoperative MRI obtained after the laser fiber had been removed. This resulted in transient hemiparesis that fully resolved on follow-up.

The mean follow-up time was 1.0 year (range 1 month to 2.86 years). It should be noted that the Engel and ILAE classifications are not ideal for capturing the effects of a palliative procedure like corpus callosotomy on aspects such as reduction of drop attacks or seizure severity unless it also reduces seizure frequency. Given this limitation, we rated each outcome with regard to the response of the targeted seizure type (typically drop attacks) for each individual as excellent for those who had complete or nearcomplete resolution of seizures, as significant if seizure frequency or severity was reduced by a clinically significant amount, and as poor if no noticeable change in seizure type or reduction in seizure frequency was noted (Table 1).

Information for the patients in this series is summarized in Table 1. Ages ranged from 5.3 to 25.0 years. Five procedures required 1 laser fiber, 5 required 2 laser fibers, and 1 required 3 laser fibers. The length of stay ranged from 1 to 6 days, with a median of 2 days.

\section{Discussion}

\section{Review of the Literature to Date}

At the time of writing, few case reports and small case 
Roland et al.

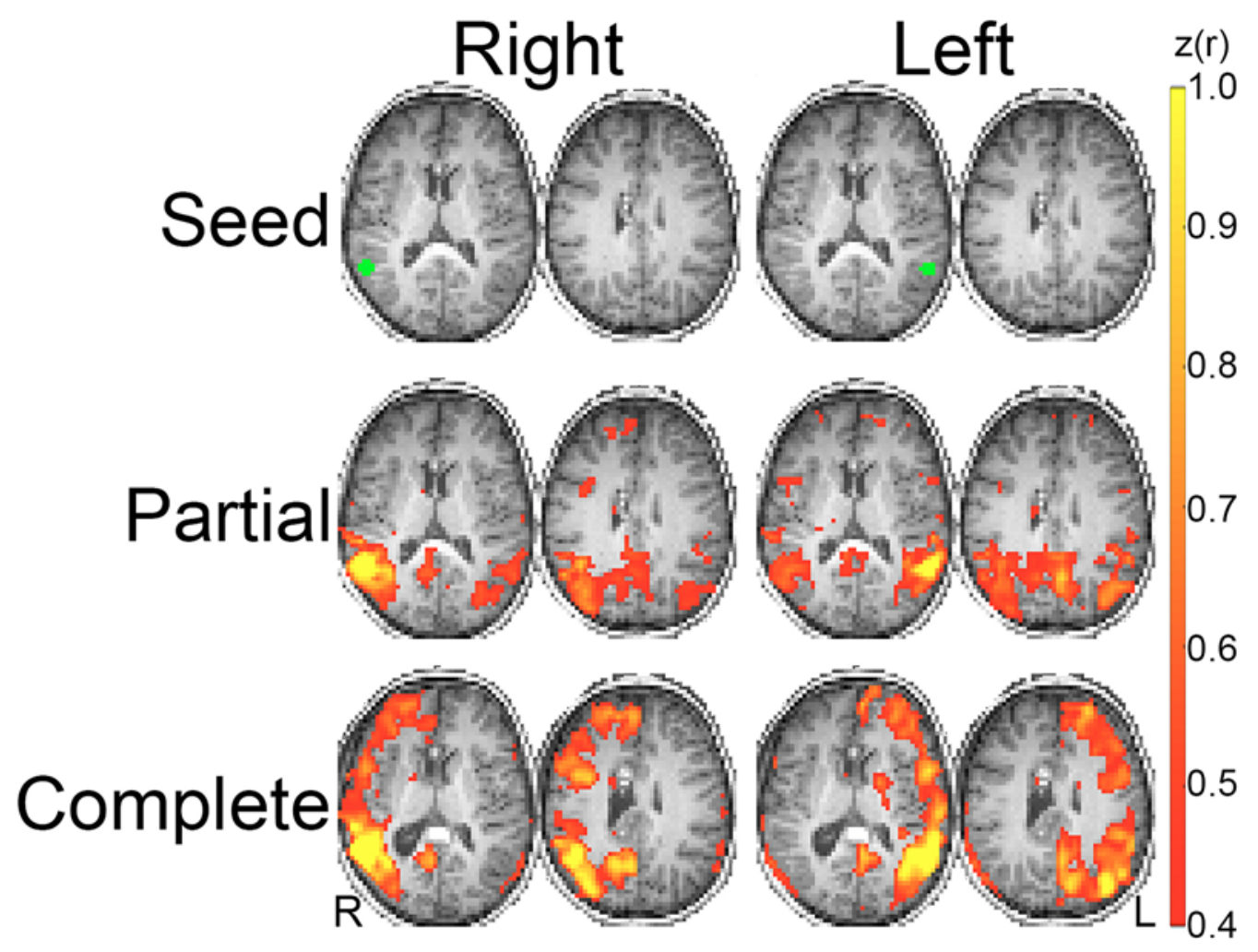

FIG. 5. Decreased interhemispheric functional connectivity after laser callosotomy. For the patient who returned after case 5 for completion of the callosotomy in case 7, we analyzed resting-state FC after each procedure. The seed locations are shown in green in the top row in the right and left hemispheres. FC maps at 2 slices in the axial plane are shown after partial LITT callosotomy (case 5) and after the second procedure for complete LITT callosotomy (case 7). Of note, after complete callosotomy, FC in the hemisphere contralateral to the seed is lost. Color intensity indicates Fisher z-transformed correlation coefficient ( $r$ ) with an arbitrary threshold of $z(r)>0.4$ for visualization. Figure is available in color online only.

series have been published describing corpus callosotomy with LITT. Ho et al. published the first case report using LITT for ablation of the splenium of the CC for MRE in a 30 -year-old patient. ${ }^{12}$ They used a single laser fiber to ablate the residual splenium of the $\mathrm{CC}$ after a previous attempt at complete callosotomy. They reported a greater than $50 \%$ reduction in seizure frequency at the 4-month follow-up. Karsy et al. published a video abstract describing their method for performing anterior corpus callosotomy with LITT in a 17-year-old patient. ${ }^{15}$ They used 3 laser fibers to ablate the anterior two-thirds and reported a significant seizure reduction on 9-month follow-up. Tao et al. described 2 cases in which they performed an anterior two-thirds corpus callosotomy with LITT. ${ }^{31}$ They used a

TABLE 1. Patient information

\begin{tabular}{|c|c|c|c|c|c|c|c|}
\hline Case No. & Age (yrs) & $\begin{array}{l}\text { No. of } \\
\text { Fibers }\end{array}$ & $\begin{array}{l}\text { Length of Stay } \\
\text { (days) }\end{array}$ & Follow-Up (yrs) & Engel Class & Targeted Seizure Response & Seizure Onset \\
\hline 1 & 5.9 & 1 & 2 & 2.86 & IV & Poor & $13 \mathrm{mos}$ \\
\hline 2 & 6.1 & 1 & 1 & 1.73 & III & Excellent & $4 \mathrm{mos}$ \\
\hline 3 & 25.0 & 1 & 1 & 1.65 & IV & Poor & $6 \mathrm{mos}$ \\
\hline 4 & 16.1 & 1 & 2 & 0.91 & III & Significant & 3 yrs \\
\hline $5^{*}$ & 5.3 & 2 & 6 & & & & 4 yrs \\
\hline 6 & 19.9 & 2 & 3 & 0.96 & III & Significant & $1 \mathrm{yr}$ \\
\hline 7 & 5.8 & 3 & 3 & 0.88 & IV & Poor & 4 yrs \\
\hline 8 & 19.6 & 2 & 3 & 0.54 & II & Excellent & $18 \mathrm{mos}$ \\
\hline 9 & 14.9 & 2 & 4 & 0.56 & III & Significant & 4 yrs \\
\hline 10 & 14.5 & 2 & 2 & 0.27 & IV & Poor & $1 \mathrm{yr}$ \\
\hline 11 & 24.6 & 1 & 1 & 0.06 & IV & Poor & 5 yrs \\
\hline
\end{tabular}

* This patient underwent a second procedure, which is recorded in case 7. 
2-laser fiber configuration with a posterior approach to the body of the CC in both patients. Ball et al. reported using LITT for anterior corpus callosotomy in a 21-year-old male. ${ }^{3}$ They also used a $2-$ laser fiber configuration with a posterior approach to the body of the CC.

We found an anterior approach to the CC body to be more favorable than a posterior approach as described in the literature to date. The anterior approach has a shorter distance and facilitates positioning in the scanner such that the patient is not lying on the anchoring bolt and exiting fiber or placed in a nonanatomic position with the head turned laterally.

Pruitt et al. reviewed complications in their institutional experience of LITT for all indications. ${ }^{23}$ Their series included 3 procedures for corpus callosotomy with no complications. Lehner et al. provided the largest case series to date of corpus callosotomy performed with LITT in 5 patients. ${ }^{17}$ In addition, they similarly used rs-fMRI in 2 patients to qualitatively support effective functional disconnection.

Palma et al. reported 3 pediatric patients who underwent LITT for corpus callosotomy. ${ }^{22}$ They described longer case times with LITT at 5.17 hours compared with open craniotomy at 2.97 hours. They commented on potentially higher direct costs for LITT due to the disposable laser fibers with reference to unpublished data. Yet, they acknowledged that a shorter length of stay and patient comfort are improved.

Singh et al. published theoretical laser fiber trajectories that were planned with structurally normal MRI scans obtained from 10 patients. ${ }^{30}$ They suggested the potential for performing partial callosotomy with a single laser from a posterior approach and complete callosotomy with only 2 laser fibers. Of note, they relied on the directional conformation of a side-firing laser, which is placed principally in the body of the $\mathrm{CC}$ along its long axis, to ablate tangentially toward the rostrum and splenium of the CC.

Collectively, the above-mentioned case reports and case series represent a total of 15 previously published LITT procedures for corpus callosotomy. The addition of our 11 cases in 10 patients brings the total number of reported procedures to 26 .

\section{Surgical Procedure}

The technical procedure to perform LITT for epilepsy has been described a variety of ways. Some hospitals have an integrated iMRI suite, which facilitates placing and removing the fiber in the same setting as LITT. Alternatively, the LITT hardware and software can be installed in a diagnostic MRI suite. This was the method we employed for 10 of our 11 procedures. Some institutions mitigate the need to travel by placing the laser fiber in the diagnostic MRI suite using nonferromagnetic equipment. Each configuration has advantages and disadvantages that will factor differently at respective institutions.

We chose to primarily use the Visualase system for corpus callosotomy due to the availability of a small $1.65-\mathrm{mm}$ diameter laser fiber. A thin fiber is well suited for small ablation targets, such as the CC cross-sectional area. Monteris offers laser probes in 3.3-mm and 2.2-mm diameters. Larger-diameter probes may be advantageous for the treat- ment of larger lesions such as neoplasia or lesional epilepsy.

The Monteris NeuroBlate system has an optional sidefire probe, as opposed to the omnidirectional diffusion tip offered by both systems. The directional laser does not preclude some degree of omnidirectional heating but can help to conform the treatment zone in one direction. Such directionality may be advantageous for treating around a corner, such as the bend of the $\mathrm{CC}$ at the genu or splenium as suggested in a theoretical model. ${ }^{30}$

\section{Complications}

In their case series, Lehner et al. described a case with significant off-target placement for 2 of the 3 laser fibers used. This resulted in incomplete ablation of the CC. $\cdot{ }^{17} \mathrm{In}$ our series, we similarly experienced misplacement of $1 \mathrm{fi}-$ ber in case 5 (Fig. 3A). This was the only fiber of 17 total fibers placed $(0.06 \%)$ to require revision.

Case 5 also had the unfortunate experience of a small hemorrhage that occurred on removal of the laser fiber. In an institutional review of 17 LITT procedures performed for all indications, Pruitt et al. reported a similar case of hemorrhage not present on initial placement but occurred on laser fiber removal. ${ }^{23}$ They reported a normal anatomical MRI on completion of the ablation, yet a routine CT scan obtained on postoperative day 1 revealed an asymptomatic intracranial hemorrhage. One of the 5 patients undergoing LITT for corpus callosotomy in the case series by Lehner et al. experienced a hemorrhage at the cortical surface where the laser fiber was placed. ${ }^{17}$

\section{Partial Versus Complete Callosotomy}

Thus far, we have not attempted an upfront complete corpus callosotomy with LITT in a patient with a morphologically typical CC. The literature to date has included only partial callosotomy and completion of prior partial callosotomy or functional hemispherotomy. This is likely related to the learning curve and early experience thus far.

We follow the TDE intraoperatively, which grows as the fiber is advanced along the trajectory until one fiber overlaps with the path of a second fiber. The TDE is confirmed on postoperative imaging with a gadolinium-enhanced T1-weighted image. The interface where 2 ablation zones meet is demarcated by an enhancing margin (Fig. 6).

The next step will be to attempt complete callosotomy upfront in appropriately selected patients. We anticipate that a third laser fiber will be required to reach the posterior body and splenium of a morphologically typical CC. Theoretically, the use of a directional laser fiber could help achieve similar coverage with a 2-fiber configuration, as suggested by Singh et al., ${ }^{30}$ but this has yet to be demonstrated in vivo. However, the morphology of the CC is highly variable between individuals and some could be more amenable to complete ablation with fewer fibers. We illustrate such an exemplar CC that is relatively straight with very little curvature in Fig. 7A.

\section{Outcomes Compared With Open Procedure}

The success of LITT for corpus callosotomy should be judged by outcomes and safety. Chan et al. recently report- 


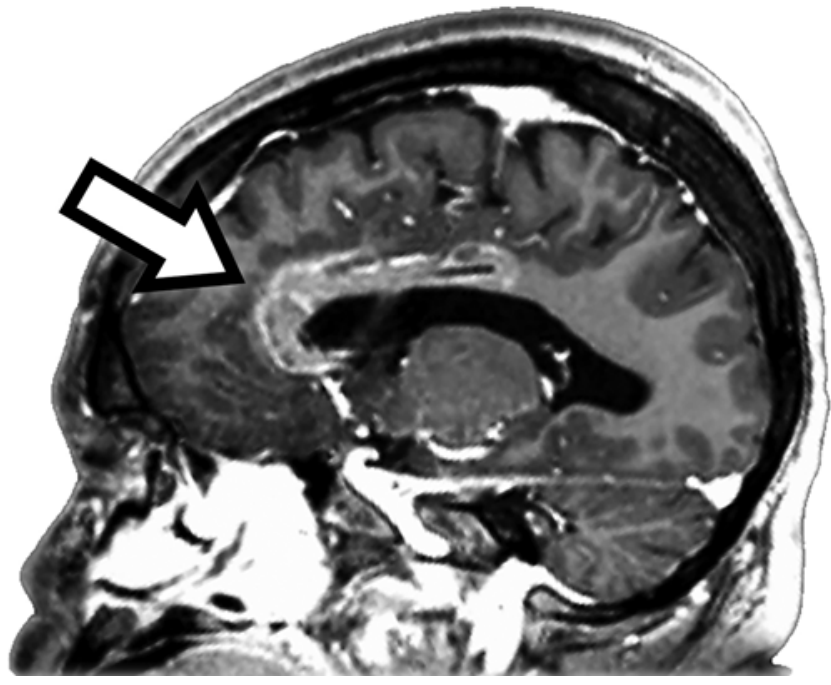

FIG. 6. Contrast enhancement after LITT callosotomy. The area of ablation is well demarcated by a rim of gadolinium contrast enhancement on a sagittal T1-weighted image. A margin of enhancement (arrow) is demonstrated at the interface where the ablation zones from the genu and body laser fibers meet.

ed the largest outcome data for open corpus callosotomy in a meta-analysis of 1742 patients across 58 studies. ${ }^{4}$ They found complete seizure freedom in $18.8 \%$ and freedom from drop attacks in 55.3\%.

Our previously published experience with open microsurgical callosotomy suggests improved seizure outcome with upfront complete callosotomy relative to anterior two-thirds only or staged anterior two-thirds followed by completion procedures if necessary. ${ }^{16}$ For this reason, we continue to offer upfront complete callosotomy with an open procedure to patients. We anticipate that certain anatomical considerations will continue to require an open surgical procedure, such as those with a high-arching or very thin $\mathrm{CC}$ (Fig. 7B). Currently, our numbers are too small and heterogeneous to reliably compare LITT and open procedures. In this series, only 5 of 11 patients underwent upfront anterior two-thirds callosotomy, of whom

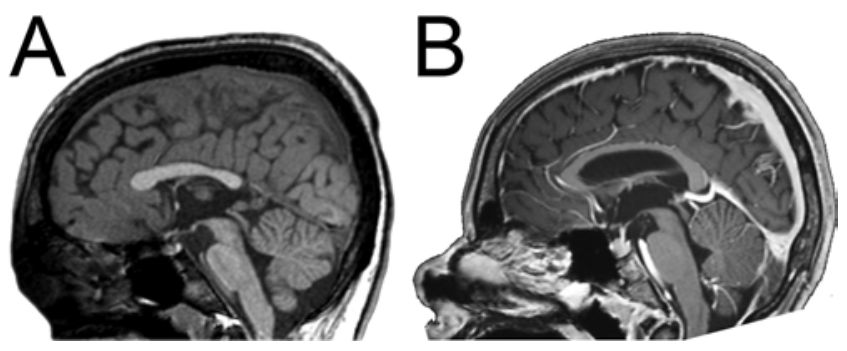

FIG. 7. Exemplar of favorable and unfavorable CC morphology for LITT. A: This CC is relatively straight and thick throughout the majority of the body. The minimal curvature at the rostrum and splenium facilitates easier targeting for LITT. B: In contrast, this CC has an atypically thin profile, which increases the risk of aberrant fiber placement. The body of the CC is high arching, as opposed to straight throughout the body segment, which makes placing a single laser fiber along the majority of the CC not possible.
3 had an Engel class of II or III, and 2 had Engel class IV. Continuing to follow the seizure response from LITT and compare to open callosotomy will be important to determine its continued role in the future.

As LITT procedures become more common, the general safety and morbidity profiles will be better understood. A trend toward shorter length of stay has been reported with LITT for epilepsy ${ }^{11,36}$ as well as tumor. ${ }^{32}$ In this series we observed a mean length of stay of 2.5 days (median 2 days). This compares favorably to our previously reported experience with open corpus callosotomy with a mean length of stay of 7-8 days. ${ }^{16}$

\section{Conclusions}

LITT appears to be a safe and effective means for performing corpus callosotomy. We believe the introduction of less invasive procedures with comparable efficacy to open procedures will lower the barrier for patients to obtain appropriate surgical evaluations for MRE. Additional data are needed to confirm equipoise between open craniotomy and LITT for corpus callosotomy.

\section{Acknowledgments}

We thank Diego Morales for assistance with study coordination and Steven Stampehl from Medtronic Inc. for assistance with obtaining the thermography video recordings.

\section{References}

1. Abou-Al-Shaar H, Brock AA, Kundu B, Englot DJ, Rolston JD: Increased nationwide use of stereoencephalography for intracranial epilepsy electroencephalography recordings. J Clin Neurosci 53:132-134, 2018

2. Asadi-Pooya AA, Sharan A, Nei M, Sperling MR: Corpus callosotomy. Epilepsy Behav 13:271-278, 2008

3. Ball T, Sharma M, White AC, Neimat JS: Anterior corpus callosotomy using laser interstitial thermal therapy for refractory epilepsy. Stereotact Funct Neurosurg 96:406-411, 2018

4. Chan AY, Rolston JD, Lee B, Vadera S, Englot DJ: Rates and predictors of seizure outcome after corpus callosotomy for drug-resistant epilepsy: a meta-analysis. J Neurosurg 130:1193-1202, 2019

5. Dierker D, Roland JL, Kamran M, Rutlin J, Hacker CD, Marcus DS, et al: Resting-state functional magnetic resonance imaging in presurgical functional mapping: sensorimotor localization. Neuroimaging Clin N Am 27:621-633, 2017

6. Dwivedi R, Ramanujam B, Chandra PS, Sapra S, Gulati S, Kalaivani M, et al: Surgery for drug-resistant epilepsy in children. N Engl J Med 377:1639-1647, 2017

7. Engel J Jr, McDermott MP, Wiebe S, Langfitt JT, Stern JM, Dewar S, et al: Early surgical therapy for drug-resistant temporal lobe epilepsy: a randomized trial. JAMA 307:922-930, 2012

8. Englot DJ, Ouyang D, Garcia PA, Barbaro NM, Chang EF: Epilepsy surgery trends in the United States, 1990-2008. Neurology 78:1200-1206, 2012

9. Graham D, Tisdall MM, Gill D: Corpus callosotomy outcomes in pediatric patients: a systematic review. Epilepsia 57:1053-1068, 2016

10. Grewal SS, Zimmerman RS, Worrell G, Brinkmann BH, Tatum WO, Crepeau AZ, et al: Laser ablation for mesial temporal epilepsy: a multi-site, single institutional series. J Neurosurg 130:2055-2062, 2019

11. Gross RE, Stern MA, Willie JT, Fasano RE, Saindane AM, 
Soares BP, et al: Stereotactic laser amygdalohippocampotomy for mesial temporal lobe epilepsy. Ann Neurol 83:575587,2018

12. Ho AL, Miller KJ, Cartmell S, Inoyama K, Fisher RS, Halpern CH: Stereotactic laser ablation of the splenium for intractable epilepsy. Epilepsy Behav Case Rep 5:23-26, 2016

13. Jalilian L, Limbrick DD, Steger-May K, Johnston J, Powers AK, Smyth MD: Complete versus anterior two-thirds corpus callosotomy in children: analysis of outcome. J Neurosurg Pediatr 6:257-266, 2010

14. Jermakowicz WJ, Cajigas I, Dan L, Guerra S, Sur S, D'Haese $\mathrm{PF}$, et al: Ablation dynamics during laser interstitial thermal therapy for mesiotemporal epilepsy. PLoS One 13:e199190, 2018

15. Karsy M, Patel DM, Halvorson K, Mortimer V, Bollo RJ: Anterior two-thirds corpus callosotomy via stereotactic laser ablation. Neurosurg Focus 44 (VideoSuppl2):V2, 2018

16. Kasasbeh AS, Smyth MD, Steger-May K, Jalilian L, Bertrand M, Limbrick DD: Outcomes after anterior or complete corpus callosotomy in children. Neurosurgery 74:17-28, 2014

17. Lehner KR, Yeagle EM, Argyelan M, Klimaj Z, Du V, Megevand $\mathrm{P}$, et al: Validation of corpus callosotomy after laser interstitial thermal therapy: a multimodal approach. J Neurosurg 131:1095-1105, 2019

18. Leuthardt EC, Guzman G, Bandt SK, Hacker C, Vellimana AK, Limbrick D, et al: Integration of resting state functional MRI into clinical practice-a large single institution experience. PLoS One 13:e0198349, 2018

19. Marashly A, Loman MM, Lew SM: Stereotactic laser ablation for nonlesional cingulate epilepsy: case report. J Neurosurg Pediatr 22:481-488, 2018

20. Medvid R, Ruiz A, Komotar RJ, Jagid JR, Ivan ME, Quencer RM, et al: Current applications of MRI-guided laser interstitial thermal therapy in the treatment of brain neoplasms and epilepsy: a radiologic and neurosurgical overview. AJNR Am J Neuroradiol 36:1998-2006, 2015

21. Mitchell TJ, Hacker CD, Breshears JD, Szrama NP, Sharma M, Bundy DT, et al: A novel data-driven approach to preoperative mapping of functional cortex using resting-state functional magnetic resonance imaging. Neurosurgery 73:969-983, 2013

22. Palma AE, Wicks RT, Popli G, Couture DE: Corpus callosotomy via laser interstitial thermal therapy: a case series. $\mathbf{J}$ Neurosurg Pediatr 23:303-307, 2018

23. Pruitt R, Gamble A, Black K, Schulder M, Mehta AD: Complication avoidance in laser interstitial thermal therapy: lessons learned. J Neurosurg 126:1238-1245, 2017

24. Rieke V, Butts Pauly K: MR thermometry. J Magn Reson Imaging 27:376-390, 2008

25. Roland JL, Griffin N, Hacker CD, Vellimana AK, Akbari SH, Shimony JS, et al: Resting-state functional magnetic resonance imaging for surgical planning in pediatric patients: a preliminary experience. J Neurosurg Pediatr 20:583-590, 2017

26. Roland JL, Hacker CD, Snyder AZ, Shimony JS, Zempel JM, Limbrick DD, et al: A comparison of resting state functional magnetic resonance imaging to invasive electrocortical stimulation for sensorimotor mapping in pediatric patients. Neuroimage Clin 23:101850, 2019

27. Roland JL, Smyth MD: Recent advances in the neurosurgical treatment of pediatric epilepsy: JNSPG 75th Anniversary Invited Review Article. J Neurosurg Pediatr 23:411-421, 2019

28. Roland JL, Snyder AZ, Hacker CD, Mitra A, Shimony JS, Limbrick DD, et al: On the role of the corpus callosum in interhemispheric functional connectivity in humans. Proc Natl Acad Sci U S A 114:13278-13283, 2017
29. Shulman GL, Pope DL, Astafiev SV, McAvoy MP, Snyder AZ, Corbetta M: Right hemisphere dominance during spatial selective attention and target detection occurs outside the dorsal frontoparietal network. J Neurosci 30:3640-3651, 2010

30. Singh H, Essayed WI, Deb S, Hoffman C, Schwartz TH: Minimally invasive robotic laser corpus callosotomy: a proof of concept. Cureus 9:e1021, 2017

31. Tao JX, Issa NP, Wu S, Rose S, Collins J, Warnke PC: Interstitial stereotactic laser anterior corpus callosotomy: a report of 2 cases with operative technique and effectiveness. Neurosurgery 85:E569-E574, 2019

32. Tovar-Spinoza Z, Choi H: Magnetic resonance-guided laser interstitial thermal therapy: report of a series of pediatric brain tumors. J Neurosurg Pediatr 17:723-733, 2016

33. Wagner K, Vaz-Guimaraes F, Camstra K, Lam S: Endoscope-assisted hemispherotomy: translation of technique from cadaveric anatomical feasibility study to clinical implementation. J Neurosurg Pediatr 23:178-186, 2018

34. Wicks RT, Jermakowicz WJ, Jagid JR, Couture DE, Willie JT, Laxton AW, et al: Laser interstitial thermal therapy for mesial temporal lobe epilepsy. Neurosurgery 79 (Suppl 1):S83-S91, 2016

35. Wiebe S, Blume WT, Girvin JP, Eliasziw M: A randomized, controlled trial of surgery for temporal-lobe epilepsy. N Engl J Med 345:311-318, 2001

36. Willie JT, Laxpati NG, Drane DL, Gowda A, Appin C, Hao $\mathrm{C}$, et al: Real-time magnetic resonance-guided stereotactic laser amygdalohippocampotomy for mesial temporal lobe epilepsy. Neurosurgery 74:569-585, 2014

37. Xu DS, Chen T, Hlubek RJ, Bristol RE, Smith KA, Ponce FA, et al: Magnetic resonance imaging-guided laser interstitial thermal therapy for the treatment of hypothalamic hamartomas: a retrospective review. Neurosurgery 83:1183-1192, 2018

38. Zhu M, Sun Z, Ng CK: Image-guided thermal ablation with MR-based thermometry. Quant Imaging Med Surg 7:356368,2017

\section{Disclosures}

The authors report no conflict of interest concerning the materials or methods used in this study or the findings specified in this paper.

\section{Author Contributions}

Conception and design: Roland, Smyth. Acquisition of data: Roland, Smyth. Analysis and interpretation of data: Roland, Smyth. Drafting the article: all authors. Critically revising the article: all authors. Reviewed submitted version of manuscript: all authors. Approved the final version of the manuscript on behalf of all authors: Roland. Administrative/technical/material support: Smyth. Study supervision: Smyth.

\section{Supplemental Information \\ Videos}

Video 1. https://vimeo.com/366225180.

\section{Correspondence}

Jarod L. Roland: University of California, San Francisco, CA. jarod.roland@ucsf.edu. 